\title{
Recurrent Prolapsed Giant Uterine Polyp Comprised of Adenomyotic Cysts
}

\author{
Vaseem Ali ${ }^{1}$, Jaou-Chen Huang ${ }^{1}$, Alyaa Al-Ibraheemi ${ }^{2}$, Jing Liu ${ }^{2 *}$ \\ ${ }^{1}$ Department of Obstetrics, Gynecology \& Reproductive Sciences, University of Texas Medical School at Houston, Houston, USA; \\ ${ }^{2}$ Department of Pathology and Laboratory Medicine, the University of Texas Medical School at Houston, Houston, USA.

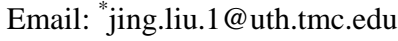

Received May 27 $7^{\text {th }}$ 2011; revised July $11^{\text {th }}, 2011$; accepted August $20^{\text {th }}, 2011$.

\begin{abstract}
The commonest pathologic diagnosis of large prolapsed polyps is leiomyoma. Benign or malignant adenomyomatous polyps follow. Adenomyotic cyst is a rare form of adenomyosis. To our knowledge, a recurrent prolapsed giant uterine polyp comprised of adenomyotic cysts and with different pathogenesis from the original polyp has not been reported in the literature. This case report describes a 29 year old woman with meno/metrrorrhagia, who was found to have a large recurrent uterine polyp prolapsed into the vagina at two and a half years after removal of an initial large uterine polyp. The initial polyp was a large uterine leiomyoma protruding through cervix. The recurrent giant polyp was comprised of adenomyotic cysts. Thus, this case report demonstrates that a prolapsed giant polyp of the uterine corpus can be caused by enlarged adenomyotic cysts inside the polyp. The pathogenesis of a recurrent uterine polyp may be different from that of the initial polyp.
\end{abstract}

Keywords: Giant, Prolapsed, Uterine Polyp, Recurrent, Adenomyotic Cyst

\section{Introduction}

Large cervical or uterine prolapsed polyps are rare [1,2]. Radiological and clinical examinations can help with distinguishing cervical polyps from the polyps of uterine corpus. Giant polyps of uterine corpus include fibroid polyp, uncommonly adenomyomatous polyp, mixed Müllerian tumor, adenomyosarcoma etc. A giant uterine polyp caused by adenomyotic cysts prolapsed into the vagina has not been reported in the literature while it can be associated with dysmenorrheal [3-5].

\section{Case Report}

A 29-year-old Asian female presented to a gynecology clinic with the chief complaint of heavy menstrual cycles, lower abdominal pain and dyspareunia.

Past gynecological history was significant for heavy painful menstrual cycles lasting for 7 days every 30 days. A laparoscopy in two and a half years ago revealed a retroverted uterus with a large uterine polyp protruding through cervix and normal tubes and ovaries. The large polyp ( $6 \mathrm{~cm}$ in greatest dimension) was pedunculated and was removed vaginally at the same time. The histology showed it to be an edematous leiomyoma.

Two years later the patient's symptoms recurred. She was found to have uterine fibroids and then had another hysteroscopic removal of uterine fibroids.

However, the symptoms were persistent. A follow-up sonohysterogram six months later showed a complex intrauterine fluid filled mass suspicious for non communicating uterine horn versus a polyp. A MRI of the pelvis with and without gadolinium contrast confirmed a septated calcified intrauterine mass filled with fluid. The diagnosis of a degenerating leiomyoma was made. On bimanual pelvic examination, the patient had a large intrauterine mass with $3 \mathrm{~cm}$ cervical dilation. This mass prolapsed spontaneously into the vagina on the follow up visit four weeks later. The clinical differential diagnosis included a recurrent prolapsed uterine fibroid polyp versus chronic uterine inversion. A transvaginal ultrasound demonstrated a $5.0 \times 6.0 \times 4.0 \mathrm{~cm}$ bi-lobed vaginal mass with two large cystic areas inside it (Figure 1(a)) and a thick stalk arising from the anterior wall of the uterine corpus (Figure 1(b)). A polypectomy was performed. As it was difficult to access the pedicle, the polyp was debulked by coring with knife. Dark chocolate colored material was drained from the cystic area of the polyp. Three endoloopa of O Vicryl ${ }^{\circledR}$ were applied to the pedicle. The polyp was removed by excising the pedicle distal to 


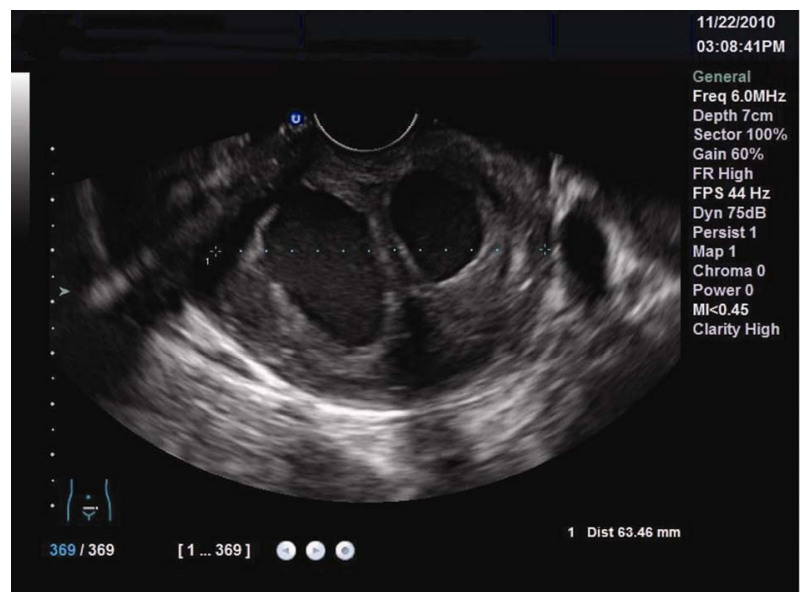

(a)

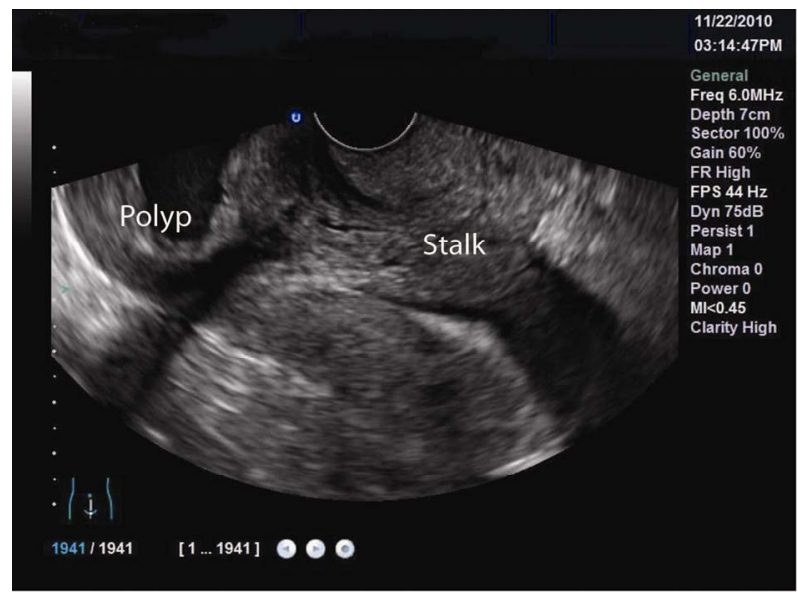

(b)

Figure 1. Transvaginal ultrasound showing the recurrent prolapsed uterine polyp with two large cystic areas (a) and the thick stalk arising from the anterior wall of the uterine corpus (b).

the endoloops. Histological examination revealed that the polyp was comprised of uterine wall-like tissue with inner surface (luminal surface of adenomyotic cyst) lined by endometrium (Figure 2(a)) and the majority of outer (protruding) surface with inflammatory infiltrate and granular debris (Figure 2(b)). The initial pathology impression was rudimentary uterine horn. After reviewing the patient's history, the final diagnosis is a benign uterine polyp secondary to adenomyotic cysts. After excision of this giant polyp, patient's symptoms improved.

\section{Discussion}

Adenomyosis is a condition characterized by the presence of ectopic endometrial tissue within the uterine myometrium. The pathogenesis is presumed to be due to endometrial invagination into myometrium or estrogen stimulation of Müllerian rests. Iatrogenic implantation is

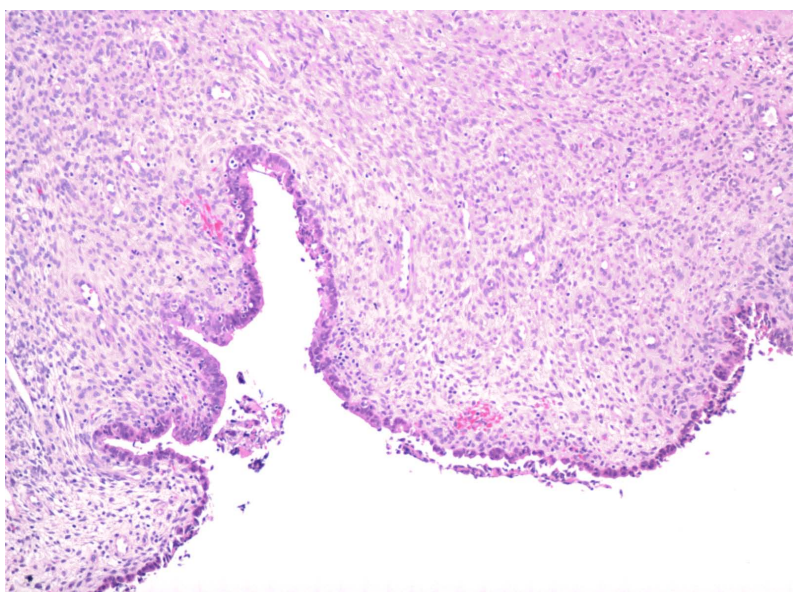

(a)

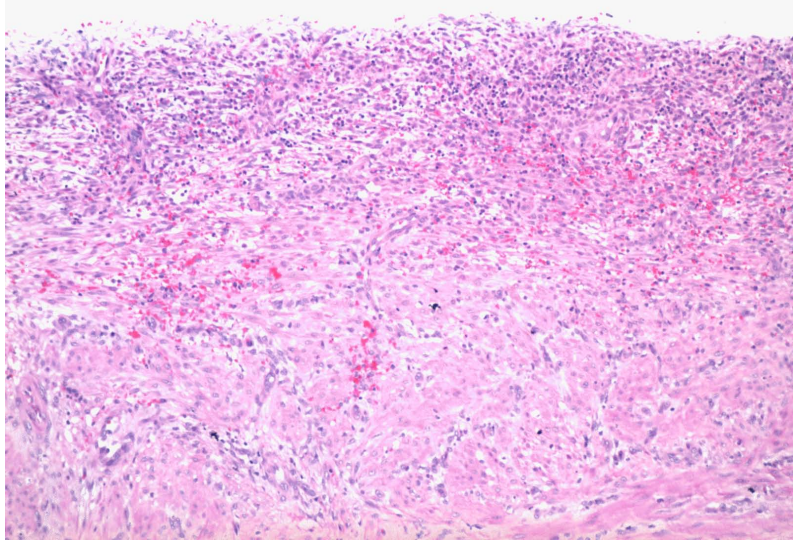

(b)

Figure 2. The recurrent giant prolapsed uterine polyp comprised of large adenomyotic cysts: (a) the luminal surface of the adenomyotic cysts showing benign endometrial epithelium and stroma and (b) the outer (protruding) surface with inflammatory infiltrate and granular debris (Hematoxylineosin stain, $\times 100)$.

also a potential mechanism in women who have undergone uterine surgery. Adenomyotic cyst is an extremely rare form of adenomyosis [6]. In this case, congenital uterine anomaly (rudimentary uterine horn) was suspected due to the polyp composed of normal appearing myometrium and endometrium before knowing the past history of this patient. However, congenital uterine anomalies are usually associated with other Müllerian duct malformations, e.g., imperforate hymen, uterine septum, and vaginal stresia/agenesis, which are usually discovered at younger age. Instead of having Müllerian duct malformations, this patient had multiple previous surgical resections for uterine fibroids. It has been documented that repeated surgical intervention is a risk factor for adenomyotic cyst [7]. The recurrent giant polyp in the 
current case can be attributable to a progressive increase in the amount of intracystic bleeding. Ruptured adenomyotic cyst of the uterus has been reported [8]. Thus, either excision of the adenomyotic cyst or hysterectomy is the treatment option. Early identification of an adenomyotic cystic lesion is essential for appropriate treatment.

\section{Conclusions}

The present case demonstrates that a recurrent prolapsed giant uterine polyp may be caused by adenomyotic cyst. A recurrent giant uterine polyp may have a different pathogenesis from the initial polyp.

\section{REFERENCES}

[1] D. Bucella, B. Frederic and J. C. Noel, "Giant Cervical Polyp: A Case Report and Review of a Rare Entity,” Archives of Gynecology and Obstetrics, Vol. 278, No. 3, 2008, pp. 295-298.

[2] V. A. Fitzhugh, G. Murphy and D. S. Heller, "Adenomyomatous Polyp of the Endometrium: A Case Report," Journal of Reproductive Medicine, Vol. 53, No. 3, 2008, pp. 231-234.
[3] M. Kamio, S. Taguchi, T. Oki, et al., "Isolated Adenomyotic Cyst Associated with Severe Dysmenorrheal," Journal of Obstetrics and Gynaecology Research, Vol. 33, No. 3, 2007, pp. 388-391. doi:10.1111/j.1447-0756.2007.00543.x

[4] M. Tamura, T. Fukaya, R. Takaya, C. W. Ip and A. Yajima, "Juvenile Adenomyotic Cyst of the Corpus Uteri with Dysmenorrheal," Tohoku Journal of Experimental Medicine, Vol. 178, No. 3, 1996, pp. 339-344. doi:10.1620/tjem.178.339

[5] M. L. Ho, V. Ratts and D. Merritt, “Adenomyotic Cyst in an Adolescent Girl," Journal of Pediatric and Adolescent Gynecology, Vol. 22, No. 3, 2009, pp. e33-e38. doi:10.1016/j.jpag.2008.05.011

[6] A. Tahlan, A. Nanda and H. Mohan, "Uterine Adenomyoma: A Clinicopathologic Review of 26 Cases and a Review of the Literature,” International Journal of Gynecological Pathololgy, Vol. 25, No. 4, 2006, p. 361.

[7] K. Koga, Y. Osuga, H. Hiroi, et al., "A Case of Giant Cystic Adenomyosis,” Fertility and Sterility, Vol. 85, No. 3, 2006, pp. 748-749. doi:10.1016/j.fertnstert.2005.11.028

[8] S. Keating, N. F. Quenville, G. W. Korn and P. B. Clement, "Ruptured Adenomyotic Cyst of the Uterus: A Case Report,” Archives of Gynecology, Vol. 237, No. 3, 1986, pp. 169-173. doi:10.1007/BF02133861 